\title{
Circular RNA circ_0003204 inhibits proliferation, migration and tube formation of endothelial cell in atherosclerosis via miR-370-3p/TGFßR2/phosph-SMAD3 axis
}

Shanchao Zhang ${ }^{*}$ (D), Guixiang Song, Jing Yuan, Shan Qiao, Shan Xu, Zhihua Si, Yang Yang, X u Xu ahd Aihua Wang

\section{Abstract}

Background: Circular RNAs (circRNAs) represent a class of non-coding RNA in mammals and tissue-specific, of which some could act as critical regulators the atherogenesis of cerebrovascular disease. However, the underlying mechanisms by wh ircRNA regulates the ectopic phenotype of endothelial cells (ECs) in atherosclerosis remain largely elusive.

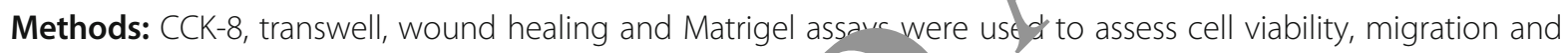
tube formation. QRT-qPCR and Immunoblotting were use to t nine targeted gene expression in different groups. The binding sites of miR-370-3p (miR-370) with To R2 O hsa_circ_0003204 (circ_0003204) were predicted using a series of bioinformatic tools, and validated asing duar. ceferase assay and RNA immunoprecipitation (RIP) assay. The localization of circ_0003204 and miR-3, N S Y vere investigated by fluorescence in situ hybridization (FISH). Gene function and pathways were enr ched th, Metascape and gene set enrichment analysis (GSEA). The association of circ_0003204 and miR-3/0 extracen,ular vesicles (EVs) with clinical characteristics of patients were investigated using multiple statisti

Results: Circ_0003204, mainly locate $d$ in the cytoplasm of human aorta endothelial cells (HAECS), was upregulated in the ox-LDL-induced HAECS. Func nally, thle ectopic expression of circ_0003204 inhibited proliferation, migration and tube formation of HAECs_exposea_ux-LDL. Mechanically, circ_0003204 could promote protein expression of TGF $3 R 2$ and its downstream pir SMAD3 through sponging miR-370, and miR-370 targeted the 3' untranslated region (UTR) of TGF $\beta R 2$ Eurtherprore, the expression of circ_0003204 in plasma EVs was upregulated in the patients with cerebral athero tero $\mathbf{s}$, anc represented a potential biomarker for diangnosis and prognosis of cerebrovascular athei

Conclusions: Co003_ could act as a novel stimulator for ectopic endothelial inactivation in atherosclerosis and a poten ial o narker for cerebral atherosclerosis.

Keywr ds: Hsa_circ_0003204, MiR-370-3p, TGFßR2, Endothelial cell, Atherosclerosis

*Correspondence: zhangshanchao2012@163.com

Department of Neurology, the First Affiliated Hospital of Shandong, First

Medical University, NO.16766 JingShi Road, Jinan 250014, Shandong, China

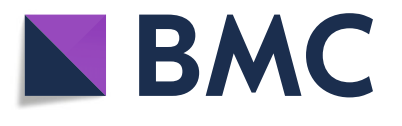

(C) The Author(s). 2020 Open Access This article is distributed under the terms of the Creative Commons Attribution 4.0 International License (http://creativecommons.org/licenses/by/4.0/), which permits unrestricted use, distribution, and reproduction in any medium, provided you give appropriate credit to the original author(s) and the source, provide a link to the Creative Commons license, and indicate if changes were made. The Creative Commons Public Domain Dedication waiver (http://creativecommons.org/publicdomain/zero/1.0/) applies to the data made available in this article, unless otherwise stated. 


\section{Introduction}

Atherosclerosis, characterized as lipid deposition and fibrous cap formation in the arterial wall, is a chronic inflammatory disease that contributes to most common vascular diseases, such as cerebral infarction, cardiovascular disease, which give rise to high mortality in aged population [1-3]. Aberrant ECs injury is regarded as one of pathological characteristics in the progression of atherosclerosis [4]. During the initiation and development of atherosclerosis, ECs are exposed to various pathogenic threats, such as ox-LDL, which display abnormal proliferation, migration and vasculogenesis that are responsible for breakdown of the integrity of endothelium to aggravate lipid deposition and fibrous cap rupture in return $[5,6]$. Besides, damaged ECs produce multiple types of cytokines which refer to interleukin, adhesion molecules, matrix metalloproteinases, etc., which participate in the atherogenesis [7]. Therefore, the study on the unknown molecular mechanisms underlying EC aberrant transformation in atherosclerosis could provide us with potential targets for reversing EC injury and clinical prevention.

Overwhelming evidences have indicated that variable microRNA alterations are associated with atherosclerotic plaque progression and regression [8,9]. For example, the expression of miR-103 is significantly upregulate a m ox-LDL-exposed HAECs, and miR-103 directly oc s PTEN expression for activating MAPK signaling $\mathrm{P}$ way, which are involved in inflammation $/$ cess an endoplasmic reticulum stress in athero creros vlaque [10]. Importantly, in addition to miRM A, other no, NAs, such as long intergenic ncRNAs an circRNAs also are involved in pathological process of aros ierosis [11, 12]. Noticeably, circRNAs, fi ned by non-sequential back-splicing of pre-mRNA tr. ipt, are widely expressed in eukaryotic and characterized by tissuespecific expression ? o omna ed with linear RNAs, circRNAs have ciarac "stic stable structure of covalently closed $/ \mathrm{s}$ witho $/$ a free $5^{\prime}$ or $3^{\prime}$ end [14]. These traits strons imply that circRNAs might serve as potential biomarkers, or human disease. Recently, growing evia inclicated circRNAs may participate in the ath gen in the way as interaction with RNA1 indi 5 proten (RBP) or miRNA sponges to alter downst m gure expressions [11, 15], for example, previous data se suggested that the circANRIL modulates ribosomal RNA maturation through binding with pescadillo homologue 1 , which, as a consequence, induces cellular proliferation inhibition and apoptosis in atherosclerosis plaque [11]. Additionally, aberrant upregulation of circCHFR in the ox-LDL-induced vascular smooth muscle cell (VSMCs) enhances the proliferation and migration of VSMCs by sponging miR-370, leading to vascular remodeling and the generation of collagen fibers
[16]. However, little is known about the functional role of circRNAs in EC aberrant phenotype in atherosclerosis. A recent study reported that increased expression of circ_0003575 by ox-LDL could block the proliferation and angiogenic ability of human umbilical vein endothelial cell (HUVEC) [17]. Although this previous study never explored the detailed mechanisms upon circRNA function underlying EC injury in depth, it provi a hypothesis that circRNAs may act as crucial regula. of aberrant EC phenotype in the ather nesis, Which, hence, is the focus of our present struy.

In the current study, we ident fied a circs, NA (circ 0003204) in HAECs, and fu er in estigated its expression and function io in model and expressive profile in the plasma $V_{s}$, from the patients with cerebral ather ss osis. Ire found that circ 0003204 may act as a o peting endogenous RNA (ceRNA) to r gu. e TGEpR2 and its downstream gene by deco, $\quad$ R-370, leading to inhibition of proliferation, $\mathrm{ration}$ and capillary-like formation of ox-L xposed HAECs. Besides, our study showed that $/ \mathrm{CH}_{1}$, _0003204 in plasma EVs served as a potential biomarker for diagnosis and prognosis of $\mathrm{Ce}_{1}$ al atherosclerosis.

\section{terials and methods}

\section{Study population}

From April to December 2018, a total of 67 individuals were recruited from the First Affiliated Hospital of Shandong First Medical University, and categorized into cerebral atherosclerosis group $(n=35 ; 18$ males and 17 females; mean age $=65.18 \pm 11.93)$ and control group $(n=$ 32; 17 males and 15 females, mean age $=60.76 \pm 10.91$ ). The inclusion criteria of patients was described as previous study with a little modification [18]. Briefly, all the patients were examined by MRI or CT to exclude any previous history of stroke. Atherosclerosis and angiostenosis were assessed through examination of cerebrovascular TCD/MRA/CTA. Patients with cerebral atherosclerosis and vascular stenosis greater than or equal to $50 \%$ were included in the cerebral atherosclerosis group. The subjects without atherosclerosis or vascular stenosis less than $50 \%$, were selected as control group. We excluded all subjects that could be diagnosed as these disorders following below, such as severe heart disease, stroke, intracranial hemorrhage, dissection, vasculitis, severe infections, nephrosis disease, liver disease, thrombotic diseases and tumors. Baseline characteristics were documented at the time of admission, including age, gender, history of diabetes mellitus (DM), smoking, drinking and hypertension. Laboratory parameters were also derived, including triglyceride (TG), total cholesterol (TC), low-density lipoproteincholesterol (LDL-C), high-density lipoprotein cholesterol (HDL-C), homocysteine level and Lipoprotein 
phospholipase A2. Hypertension was defined as resting systolic blood pressure $\geq 140 \mathrm{mmHg}$ and/or diastolic blood pressure $\geq 90 \mathrm{mmHg}$. Diabetes was defined as fasting blood glucose $\geq 7.0 \mathrm{mmol} / \mathrm{L}$ or a diagnosis of diabetes needing diet. Individuals who formerly or currently smoked more than 6 months or daily smoked more than 20 cigarettes were defined as smokers. Excessive drinking is defined as drinking alcohol more than $25 \mathrm{~g} /$ day for adult males and more than $15 \mathrm{~g} /$ day for adult females. These subjects were followed until time to event or, in the case of no event, until August 2019. The end event was a composite outcome of stroke, transient ischemic attack (TIA), major vascular events and mortality. The diagnosis of stroke and TIA was defined according to the American Heart Association/American Stroke Association guideline [19]. The mortality was defined as cerebrovascular death. The follow-up rate was $91 \%$. This study was reviewed and approved by Institutional Review Board of the First Affiliated Hospital of Shandong First Medical University, and patient consent was acquired prior to the initiation of experiment.

\section{Cell culture}

HAECs was purchased from ScienCell (Carlsbad, CA, USA). The cells were cultured in endothelial ceil medium supplemented with $10 \% \mathrm{FBS}, 1 \%$ endothelial cell growth supplement (ECGS), $100 \mathrm{IU} / \mathrm{ml}$ penicill: 0.) $\mathrm{mg} / \mathrm{ml}$ streptomycin (ScienCell) at $37^{\circ} \mathrm{C}$ in a tumia. atmosphere of $5 \% \mathrm{CO} 2$. Confluent HAEC re main, tained for $48 \mathrm{~h}$ with or without the presence $\mathrm{o}_{1}$ v-LDL $(50 \mu \mathrm{g} / \mathrm{mL}$; Beijing Solarbio Life Scie ice Compar $y)$ for further study.

\section{EVs isolation}

EVs were extracted from HAEC Cha alture medium or plasma samples using Exo(buick precipitation kit (SBI, System Bioscie s, I lountan view, CA) according to the manufactyor's tructions. Briefly, the culture medium and $p \%$ a were lawed on ice and centrifuged at $3000 \times \mathrm{g}$ for $1 \mathrm{~s}$ in and $10,000 \times \mathrm{g}$ for $30 \mathrm{~min}$. For plasma $\angle V$ isolation, $250 \mu \mathrm{l}$ of the supernatant was mixed $>(7)$ of the ExoQuick precipitation reagent and cuba $4^{\circ} \mathrm{Cfor} 30 \mathrm{~min}$, followed by centrifugaon $3000, \mathrm{~g}$ for $10 \mathrm{~min}$. For the isolation of EVs in ce meunm, an Amicon Ultra Centrifugal Filter Unit (100 ar, Millipore) was used to concentrate the supernatanit. The ultrafiltration supernatant was mixed with the ExoQuick precipitation reagent at the ratio of 5:1, and incubated at $4{ }^{\circ} \mathrm{C}$ overnight, followed by centrifugation at $1500 \times \mathrm{g}$ for $30 \mathrm{~min}$. The EV pellet was subsequently resuspended in $200 \mu \mathrm{l}$ phosphate buffered saline (PBS). This isolation method has been well validated with other techniques including electron microscopy [20]. EV concentrations and size distribution were measured by nanoparticle tracking analysis (NTA) (NanoSight, NanoSight Ltd., UK).

\section{CCK-8 proliferation vitality assay}

Cell viability was measured using the Cell Counting Kit 8 (Dojindo, Shanghai, China) according to the ma ufacturer's instructions. HAECs were seeded into 96-well plates at a density of $5 \times 10^{3}$ cells/well at $37^{\circ} \mathrm{C}$. e cell viability was measured at the time point using plate reader (Bio-Rad) by spectrophoton v at $450 \mathrm{~nm}$.

\section{Migration assay}

The migration of HAECs was d armined using a 24well modified Boyden cha er(o, Corning). Approximately $5 \times 10^{4}$ cell 2 in 0 . । serum-free medium were added in the ur po hambe) $0.6 \mathrm{ml}$ medium with $10 \%$ FBS was seeded in the vomer chamber as a chemoattractant. Followin. $24 \mathrm{~h}$ of/ncubation, the cells on the lower side of $\mathrm{t}$ c w were fixed in $4 \%$ paraformaldehyde for $20 \mathrm{~m}$ and dyed with $0.1 \%$ crystal violet staining $\mathrm{s}$ an (Beyotime, Nantong, China) for $10 \mathrm{~min}$, and then veresunted and photographed in five representative fislds. All experiments were repeated three tir. independently.

\section{ur.d healing assay}

C€1 motility was assessed by performing a woundhealing assay. Cells were cultured in 6 -well plates $(5 \times$ $10^{4}$ cells per well). At $80-90 \%$ confluence, the monolayer of cells was scratched using a sterile $200 \mu \mathrm{L}$ tip, and then, cells were cultured under standard conditions for $24 \mathrm{~h}$. Following several washes, recovery of the wound was captured at 0 and $24 \mathrm{~h}$ in a phase contrast microscope. All experiments were carried out in triplicate.

\section{Tube formation assay}

Capillary-like network formation was performed to detect the angiogenic ability of HAECs. Briefly, HAECs were seeded at a density of $2 \times 10^{4}$ on 96-well plates coated with $60 \mu \mathrm{L}$ Matrigel (BD Bioscience). Being cultured for $48 \mathrm{~h}$, the average number of capillary-like branches was counted in 5 random microscopic fields with a computer-assisted microscope.

\section{Plasmid, siRNAs and miRNA mimic and inhibitor}

Plasmid of circ_0003204 overexpression, siRNA targeting circ_0003204 and non-specific negative control were purchased from RiboBio (Guangzhou, China). The microRNA mimics/inhibitor and corresponding negative control for miR-370-3p as well as TGFßR2 siRNA were also purchased from RiboBio. The sequences of circ 0003204 siRNA, TGF $\beta 2$ siRNA and its negative control were shown in Additional file 1: Table S1. HAECs were 
planted in 6-well plates $24 \mathrm{~h}$ prior to circ_0003204 vector, miR-370 mimic or inhibitor transfection with $50-60 \%$ confluence, and then were transfected using Lipofectamine $^{\text {rax }}$ RNAiMax (Invitrogen) according to the manufacture instructions.

\section{Sanger sequencing}

The amplification products were inserted into a $\mathrm{T}$-vector for Sanger sequencing to determine their full-length. The primers were synthesized in RiboBio, and Sanger sequencing was performed by Biorui (Beijing, China).

\section{Quantitative real-time PCR (qRT-PCR)}

Total RNA was extracted extracted from EVs or cell samples using TRIzol (Thermol Fisher Scientific, MA, USA) and reverse transcription was performed using miScript II RT Kit (Qiagen, MD, USA) and cDNA amplification using the SYBR Green Master Mix kit (Takara, Otsu, Japan). The reverse transcription of circRNAs were performed using a HiScript Q RT SuperMix for qPCR Kit (Vazyme, Naijing, China) and quantified using SYBR Green Real-time PCR Master Mix. The nuclear and cytoplasmic fractions were isolated using NE-PER Nuclear and Cytoplasmic Extraction Reagents (Thermol Fisher Scientific, MA, USA). All of the primers were synthesized by RiboBio and listed in Additional file 1: Table S1.

\section{Western blot}

Proteins were extracted from EVs and celis us. RIPA Lysis and Extraction Buffer (Therm, Fisher Scly ntific, MA, USA) containing Protease/Ph sphatase Inhibitor Cocktail (Abcam, Cambridge, MA, U U. The extracted proteins were separated in 10\% $\mathrm{CDS}$-polyacrylamide gel, and then transferred to in mos n-P membranes (Merck Millipore, Da adt, Germany). The membranes were blocked ith $0 / \mathrm{w} / \mathrm{v}$ Bovine Serum Albumin (Sigma-Aldri $M$ USA), followed by incubation overnight wit/ rimary htibodies as follows: antiFLOT-1 (1:1000, ' Signaling Tech, MA, USA), antiCD63 (1.2000, Abca, 1), anti-TGS101 (1:1000, Abcam), anti-Te R- (1 1000, Abcam), anti-SMAD3 (1:1000, Ce ${ }^{1-}$ igna. $T$ ech) and anti-phosph-SMAD3 (1:1000, ell gnalin, Tech). The membranes were then incub. wru secondary antibodies (1:2000, HRP-linked anti- Sbit IgG, Cell Signaling Tech), and digital images were visualized with the use of an Immobilon Western Chemiluminescent HRP substrate (Millipore, Darmstadt, Germany).

\section{Actinomycin D and RNase R treatment}

Transcription was inhibited by the addition of $2 \mathrm{mg} / \mathrm{ml}$ Actinomycin D or DMSO (Sigma-Aldrich, St. Louis) as the negative control. Total RNA $(5 \mu \mathrm{g})$ was incubated for
$30 \mathrm{~min}$ at $37^{\circ} \mathrm{C}$ with $4 \mathrm{U} / \mu \mathrm{g}$ of RNaseR (Epicentre Biotechnologies). After treatment with Actinomycin D and RNase R, the expression levels of USP36 mRNA and circ_0003204 were detected by qRT-PCR.

\section{FISH analysis}

HAECs cultured on coverslips were fixed wit $4 \% \mathrm{PFA}$ for $10 \mathrm{~min}$ and incubated in PBS overnight $4^{\circ} \mathrm{C}$ followed by processing to detect circ_0 03204 or $\mathbb{R}$ 370 expression. Next, the cells were pen abilize 1 with $0.5 \%$ Triton X-100 in PBS for $15 \mathrm{r}$ n. After $\mathrm{h}$ dration with 70, 95 and $100 \%$ ethanol $\mathrm{f} 5 \mathrm{~min}$, hybridization buffer containing a Cy3-la'elec virc_003204 probe (RiboBio, Guangzhou, China, d a 11 C-labeled miR370 probe (RiboBio, $G$ ingzhou, China) was heated to $88^{\circ} \mathrm{C}$ for $5 \mathrm{~min}$ and $\mathrm{c}$ ipp onto the coverslips, followed by hybridization $37^{\circ} \mathrm{C}$ ernight in a dark moist chamber. The ext ay, the coverslips were washed three times in $2 \mathrm{X} \mathrm{SSO}$ te time at $42^{\circ} \mathrm{C}$, the rest at room tempture The sis $1 / s$ of the probes were detected by Fluorescein Situ Hybridization Kit (RiboBio, Guangzhou Chana) according to the manufacturer's inctw...ctions. Then, the coverslips were washed three times wit BS and incubated with DAPI (Santa Cruz Biotecholog) for $20 \mathrm{~min}$ at room temperature to visualize in 1 . The sections were finally mounted with rubber cement. Immunofluorescence images were captured via microscopy (Leica, Germany). The circ_0003204 and miR-370 probe sequences were seen in Additional file 1: Table S1.

\section{RIP assay}

RIP was performed using a Magna RIP Kit (Millipore, Billerica, MA, USA) following the manufacturer's instructions. The abundance of miR-370 and circ_0003204 was tested using qRT-PCR. The antibodies against Ago2 and IgG used for RIP were purchased from Abcam.

\section{Luciferase activity assay}

HEK-293 T cells were seeded in 96-well plates and cultured to $50-70 \%$ confluence before transfection. The constructs containing wild-type or mutant circ 0003204-miR-370 were inserted into luciferase gene by psiCHECK-2 vector as well as TGF $\beta$ R2-miR-370 by a pmirGLO vector (Promega Corporation, Madison, WI, USA). $100 \mathrm{ng}$ of luciferase reporter vectors and $20 \mathrm{pmol}$ of miR-370 mimics/NC were transfected to $293 \mathrm{~T}$ cells for $24 \mathrm{~h}$ by Lipofectamine 2000. After $24 \mathrm{~h}$ incubation, the Promega Dual-Luciferase system was used to detect firefly and Renilla luciferase activities. The ratios of firefly to Renilla luciferase activities were calculated and repeated three times to determine relative luciferase activity. 


\section{Microarray data}

The gene expression profiles of GSE13139, GSE28829, GSE34645, GSE34644 and GSE34646 were downloaded from Gene Expression Omnibus (GEO, http://www.ncbi. nlm.nih.gov/geo). GSE13139 and GSE28829 were performed on GPL570: [HG-U133_Plus_2] Affymetrix Human Genome U133 Plus 2.0 Array while GSE34645, GSE34644 and GSE34646 on GPL15053: Applied Biosystems Taqman Array Rodent MicroRNA Cards v2.0. We extracted part of data from GSE13139 for further analysis, including 3 sample of ox-LDL treatment and 3 control samples.

\section{Data preprocessing and differently expressed gene (DEG) screening}

The downloaded platform and series of matrix files were converted using the $\mathrm{R}$ language software and annotation package. The ID corresponding to the probe name was converted into an international standard name for genes (gene symbol). Gene differential expression was performed using the limma package in $\mathrm{R}$, with treated samples verse untreated ones. Multiple testing correction was done to control the overall error rate using the Benjamini-Hochberg false discovery rate (FDR). An FDR $<0.05$ and a $\mid \log _{2}$ Fold Change (FC) $\mid>2$ were used as the cut-off criterion to identify the final DEGs.

\section{Bioinformatics analysis}

We identified the predicted miRNAs ta/s ing circ, 0003204 using a bioinformatic programs. Ci teractome (http://circinteractome.nia.nih gov). The overlapped target microRNAs of signi cantly upregulated genes from GSE13139 and GSE28 wer predicted using combination of Targetso (http://targetscan.org), miRDB (http://mirdb.org) and $\mathrm{m} / \mathrm{s}$ la (http://microrna.org). GSEA (http://on vare.l roadinstitute.org/gesa/) was performed to rest ate Kyoto Encyclopedia of Genes and Genones (. GG) pathways of upregulated gene expression GSE1. 39. Selected enriched pathways had a rela. FDR $<0.25$ and $P<0.005$. Gene Ontolog (GO) and ,EGG pathways analysis were performea o dict the potential functions of genes associa wit wir_0003204 and upregulated genes with $\mathrm{g}_{2} \mathrm{~F}_{\mathrm{i}}>2$ a d $\bar{P}$-value $<0.05$ in GSE13139 using the N. iscap bioinformatics tool (http://metascape.org). Only -rms with P-value $<0.05$, minimum count of 3 , and enrichment factor of $>1.5$ were considered as significant. A subset of enriched terms was selected and rendered as a network plot to further determine the relationship among terms, where terms with similarity of > 0.3 were connected by edges. Protein-protein interaction enrichment analysis was performed using the following databases: BioGrid, InWeb_IM, and OmniPath. Further, Molecular Complex Detection (MCODE) algorithm was applied to identify densely connected network components. Topology analysis was used to analyze the connectivity of the nodes in the PPI network to obtain a higher degree of key nodes. The hub genes were selected as 'degree $>6$ ' for further analysis. Functional enrichment analysis of each module wa performed using Metascape, with a significance thr shold of $P<0.01$.

\section{Statistical analysis}

Statistical analyses were carried out Dy us SPS 17.0 (IBM, SPSS, Chicago, IL, USA) and Graph $r$ ad Prism 7.0. All continuous variables wer sxpressed as mean \pm standard deviation (SD) or $1 \mathrm{~h}$, lian rquartile range). The chi-square test or isher s vact test was used to express categorical $\mathrm{a}_{\text {. }}$ les. T, vo treatment groups were compared by the un ired Students t test. Nonnormallydistribate data were compared using MannWhitney U uskal-Wallis test. Statistical difference betwee hree or more were determined by a one-way rasis or variance. Multivariable logistic regression anoiys, was performed while evaluating the relationship between cerebrovascular atherosclerosis an lated risk factors. The predictive function for distingu hing cerebral atherosclerosis and control group characterized by ROC, and area under ROC curve $(A, C)$ was calculated for assessing the diagnostic perrormance of selected markers. The Spearson's correlation coefficient analysis was used to analyze the correlations. Event-free curves were analyzed with the Kaplane-Meier method and log-rank test. $P<0.05$ was considered statistically significant.

\section{Results \\ Identification and characteristics of circ_0003204 in HAECS}

Mounting evidence shows that circRNAs as a novel type of ncRNAs could sponge miRNAs, regulate gene transcription and interact with RBPs involved in atherosclerosis [21]. Microarray analysis of circRNA in previous study revealed that circ_0004543 and circ 0003204 expression in HUVECs are significantly increased with treatment of ox-LDL [17], but it remained unclear for these two circRNAs level in oxLDL-treated HAECs. QRT-PCR analysis indicated that treatment of HAECs by $50 \mu \mathrm{g} / \mathrm{ml}$ ox-LDL for $24 \mathrm{~h}$ could promote circ_0003204 expression in HAECs rather than circ_0004543 (Fig. 1a). Subsequently, we noted that circ_0003204 (chr17:76,798,405-76,800, 060) is derived from exon 16 and 17 regions within ubiquitin specific peptidase 36 (USP36) locus (Fig. 1b). The genomic position revealed that the 16th and 17th exons from the USP36 gene are intermediated by long introns (Fig. 1b). Compared with the linear USP36 


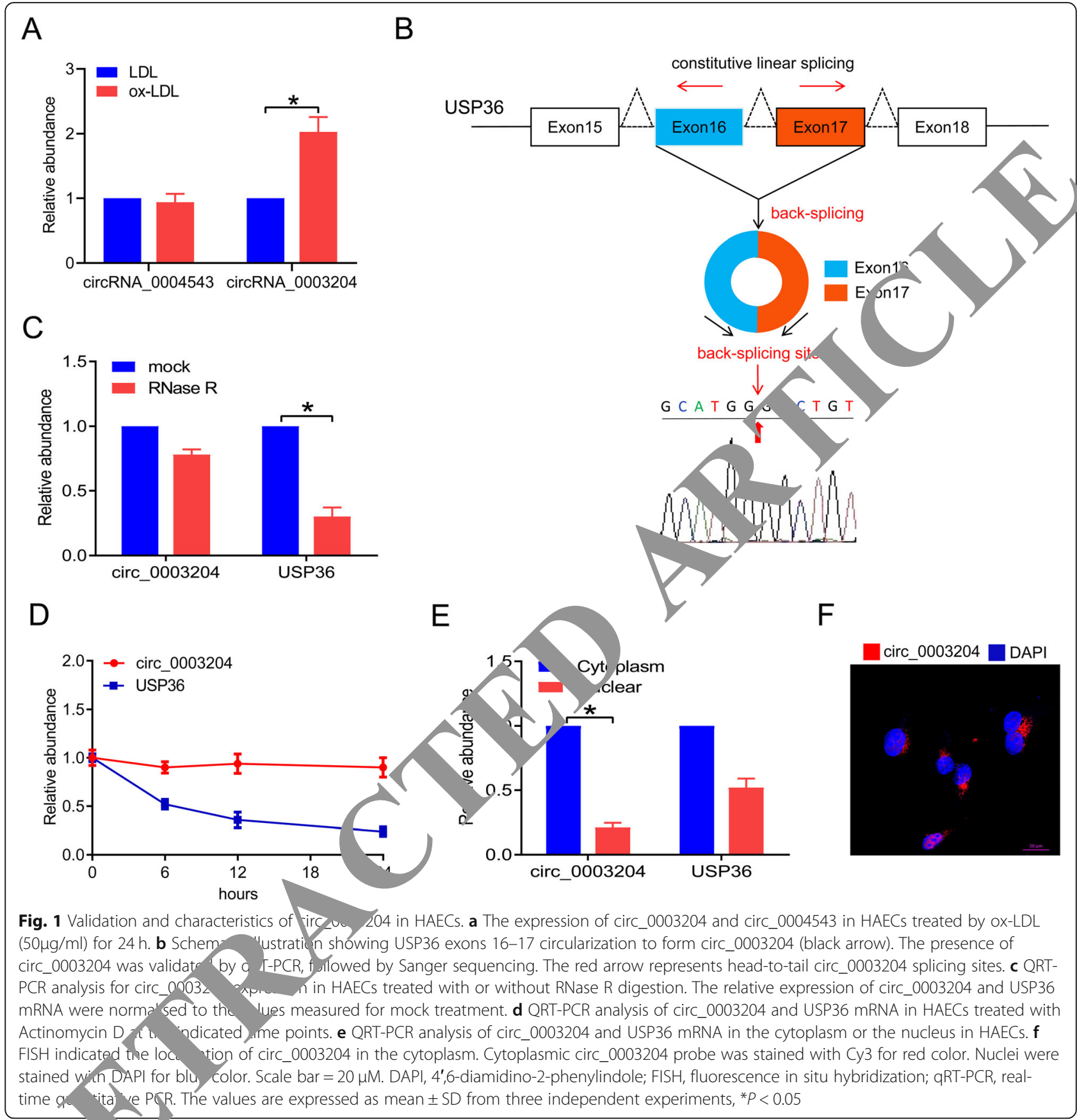

base on the qRT-PCR analysis, circ 0003204 had a resistant ability against digestion induced by RNase $\mathrm{R}$ exonuclease, indicating that circ_0003204 harbors a loop structure (Fig. 1c). We next observed the stability and localization of circ_0003204. After treatment with Actinomycin D, an inhibitor of transcription at the indicated time points, total RNA was separated from HAECs. QRT-PCR analysis showed that the transcript half-life of circ_0003204 exceeded 24. h, while that of linear USP36 displayed about $6 \mathrm{~h}$ in HAECs (Fig. 1d), indicating that circ_0003204 is highly stable in HAECs. Cytoplasmic and nuclear RNA analysis of qRT-PCR uncovered that circ 0003204 was preferentially localized in the cytoplasm in HAECs (Fig. 1e). Furthermore, FISH was used to assess circ_0003204 localization in HAECs showing that circ_0003204 (red fluorescent distribution) was mainly localized in the cytoplasm of HAECs (Fig. 1f). 


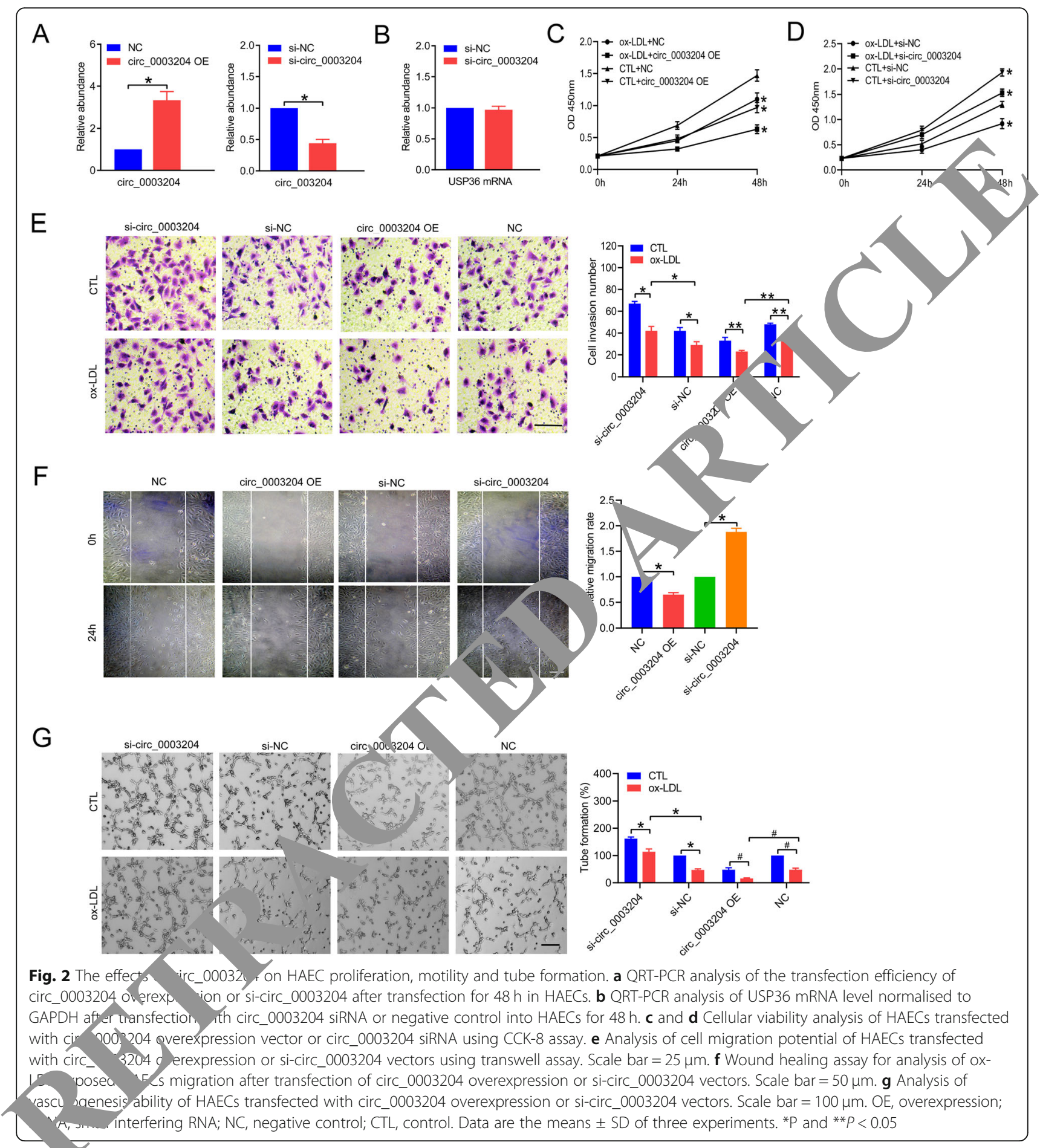

Collectively, these results suggested that circ_0003204 is a highly stable and cytoplasmic circRNA derived from the USP36 gene locus.

\section{Circ_0003204 inhibits the HAECs proliferation, migration and tube formation in vitro}

To identify the biological functions of circ_0003204 in the regulation of HAECs phenotype, we transfected
HAECs with circ_0003204 overexpresson (OE) vector and small-interfering RNA (siRNA), and then examined the expression level of circ_0003204 (Fig. 2a). Then we found that knockdown of circ_0003204 had little effect on USP36 mRNA level in HAECs (Fig. 2b). Subsequently, cell viability assay was carried out to reveal that overexpression of circ_0003204 inhibited growth of HAECs, while knockdown of circ_0003204 reversed 
the repressive effects of circ_0003204 overexpression (Fig. 2c and d). Then, transwell and scratch assays demonstrated that circ_0003204 OE could aggravate low mobility of ox-LDL- treated HAECs while circ_0003204 knockdown alleviated impaired motility of HAECs oxLDL caused (Fig. 2e and f), which was analogous to the impact of circ_0003204 on control HAECs (Fig. 2e). In addition, we dissected capillary network formation of ox-LDL-treated HAECs as well as control treatment, which uncovered that circ_0003204 acted like antagonist against tube formation of HAECs (Fig. 2g). Taken together, our results implied the involvement of circ 0003204 in the regulation of HAECs ectopic phenotype ox-LDL caused.

\section{Circ_0003204 acts as a miRNA sponge for miR-370}

Given that circ_0003204 played a critical role in the regulation of HAEC phenotype, we thus uncovered the underlying mechanisms for circ_0003204. We first downloaded microarray data from GEO datasets (GSE13139) and performed bioinformatic analysis of gene profiles referred to ox-LDL-treated HAECs. The heating cluster map revealed significant changes of DEGs in ox-LDL-treated HAECs compared with their expression in the sham group (Fig. 3a). Figure 3b yas indicative of normalization of GSE13139. The va ation of gene expression between the sham and ox- $\mathrm{LD}_{1}$ eated was shown in volcano and scatter plots in Fig. 3c a da Of these genes, 88 with $\log _{2} \mathrm{FC}>2$ an $P<0.0$ - were considered to be significantly upre ourated, $\mathrm{d} / 8$ with $\log _{2} \mathrm{FC}<-2$ and $\mathrm{P}<0.05$ were s gnificantly downregulated. We then performed GO a pathyay analysis of these 88 upregulated genes spu. We the potential functions of upregulated ciro TAs as indicated in Additional file 1: $\mathrm{Fi}, \mathrm{u}_{\mathrm{L}} \mathrm{S} 1$, for instance, one of the enriched KEGG for the up ulated genes, cAMP signaling pathway, has $\mathrm{n}$ confirmed to be involved in the progression of osis [22]. In the one hand, it is

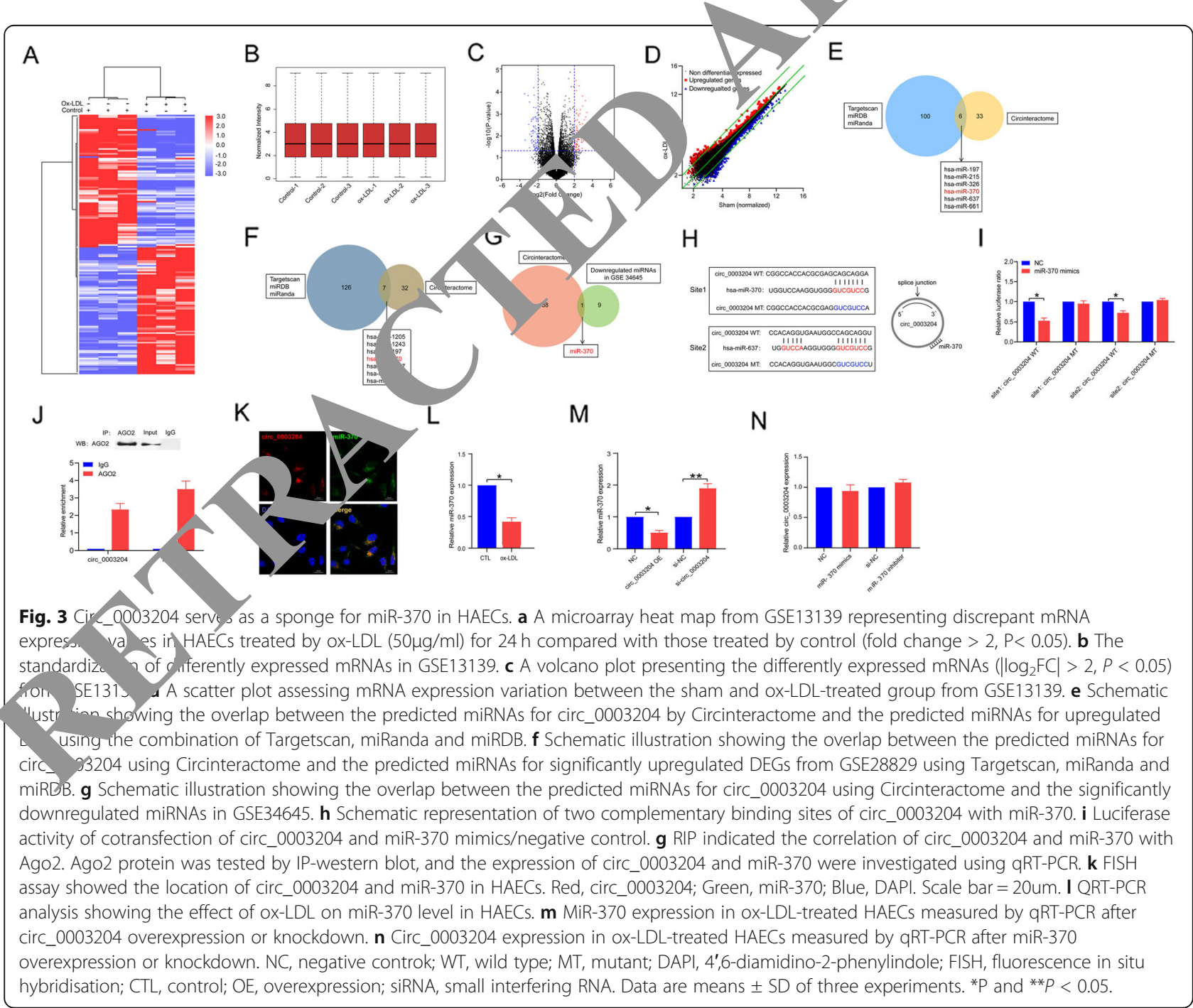


well-established that circRNAs could act as an endogenous RNA sponge to interact with miRNAs and influence the expression of targeted genes [23]. We therefore investigated which miRNAs circ_0003204 sponges. Bioinformatics programme circinteractome predicted 39 miRNAs which could bind to the recognition seed in circ_0003204 sequence, and circ_0003204/39 downstream miRNAs/mRNA interaction network was shown in Additional file 1: Figure S2. Subsequently, the combination of Targetscan, miRanda and miRDB predicted 106 candidate miRNAs which could bind to 3' UTR of 88 significantly upregulated genes in GSE13139 (Fig. 3e). Six miRNAs were selected through overlapping these two datasets (Fig. 3e). To further investigate whether circ_0003204 was involved in the progression of atherosclerotic plaques, the upregulated DEGs in GSE28829 were used to predict 133 targeted miRNAs using Targetscan, miRanda and miRDB, and then 39 circinteractome-predicted miRNAs for circ_0003204 were overlapped with these 133 targeted miRNAs, which displayed 7 overlapped miRNAs (Fig. 3f). Subsequently, to speculate the potential functions of circ_0003204, we performed GO and pathway analysis of these 13 overlapped miRNAs (6 in Fig. 3e and 7 in Fig. 3f) and their PPI network involved in atherosclerosis (Additional file 1: Figure S3). The enriched KEGG pathways, incluantg AMPK signaling pathway and FOXO signaling $p$ wi (Additional file 1: Figure S3D), have been cornme be involved in the progression of atheros osis [2, 25]. In addition, notably, both hsa-miR-370. hsamiR-197 were enriched into the overapped miRb, As in Fig. 3e and f. Due to the miRNA cor erved property between mouse and human, the expre on le vel of both mmu-miR-370 and mmu-miR- ${ }^{-}$were rurther detected in miRNA profiles GSE34645, CiSE, 4 and GSE34646. As indicated in Additio file 1. Figure S4A and B, the expressions of $\mathrm{mm}$ niR $7 \mathrm{7n}$ is early atherosclerosis plaque significantiy feli vwn to less than $50 \%$ of nonatherosclerosis ery tissu $(P<0.05)$, but no statistical significance was to $d$ in $\mathrm{mmu}-\mathrm{miR}-197$ expression between them, despite the downregulated expression of $m m u-1, z$ in the early atherosclerosis plaque. Intrigui ${ }^{-1} \mathrm{v}$, $\mathrm{t}_{\mathrm{H}}$ expression of mmu-miR-370 and mmuiR- 7 in advanced atherosclerosis plaque remained $u_{\text {. }}$ angeu (Additional file 1: Figure S4C and D). More impo antly, miR-370 was the only candidate microRNA after overlapping circinteractome-predicted miRNAs for circ_0003204 with the significantly downregulated microRNAs in GSE34645 (Fig. 3g). These results suggested that miR-370 was involved in the early development of atherosclerotic plaque. Moreover, literature reviews of miR-370 have shown that miR-370 could be downregulated by ox-LDL dose-dependently and timedependently, which induces the increase of IL-6 and IL-
$1 \beta$ [26], whereas miR-370 overexpression had positive impact on the invasion and proliferation of HUVECs [27]. Therefore, we constructed a circ_0003204 fragment and incorporated it into downstream of the luciferase reporter gene and hypothesized that miR-370 could reduce the luciferase activity of circ_0003204 (Fig. 3h). I ciferase assay confirmed that site 1 of miR-370 wo able to result in the lower luciferase reporter activity $\mathrm{c}$ oared with site 2 , indicating that miR-370 might have po to bind with circ_0003204 (Fig. 3i), the ve fould that circ_0003204 and miR-370 were more abu nt in Ago2 pellet than in the IgG pellet (Fig 3j). Subses a uently, we performed FISH assay to reveal at circ_0003204 and miR-370 colocalized in the top (Fig. 3k). Additionally, it was demon rated + miR-370 expression in HAECs could be $\mathrm{j}$ h ed by $x$-LDL (Fig. $3 \mathrm{l}$ ). Given that circ_0003204 was ab. to bind with miR-370, we then detected the pression level of miR-370 via gain/ loss-of-function of 003204 , indicating decreased expression of $\mathrm{ml} \quad 70$ concomitant with circ_0003204 overexpre whice increased expression of miR-370 with circ_pos 3,44 inhibition (Fig. 3m), but miR-370 mimics or inhibitor had no impact on the expression of CII 003204 (Fig. 3n).

\section{${ }^{-} \beta / 2$ is a target gene of miR-370}

$\mathrm{Pr}$ evious studies have confirmed that FOXO1 was identified as functional target of miR-370, which could prohibit the invasion and proliferation of human umbilical vein endothelial cells [25]. However, little is known about which signaling pathway miR-370/FOXO1 was involved in. KEGG analysis showed that FOXO1 was enriched into pathways in cancer, AMPK signaling pathway, FOXO signaling pathway, prostate cancer and insulin resistance, etc., all of which remarkably overlapped signaling pathways of circ_0003204 (Additional file 1: Figure S3D). in addition, it was found that FOXO pathway was involved in endothelial protection against atherosclerosis [28], in which TGF $\beta R 2$, a predictive target of miR-370 by combination of Targetscan, miRDB and miRanda, was involved as well [29] (Additional file 1: Figure S5). Interestingly, TGF $\beta 22$ was found to participate in TGF $\beta$ signaling pathway [30] with which signaling pathways of circ_0003204 also shared (Additional file 1: Figure S2D). Accordingly, it may speculate that the alternation in miR-370/TGFßR2 axis might underlie the biological function of circ_0003204. To investigate this hypothesis, TGF $\beta 2$ was selected to demonstrate whether it can directly bind with miR-370. Firstly, miR-370 mimic or inhibitor were respectively transfected into ox-LDL-treated HAECs. After transfection for $48 \mathrm{~h}$, qRT-PCR and western blot analysis displayed low expression of TGF $\beta$ R2 in HAECs after miR370 mimics transfection (Fig. 4a and b), and conversely, 


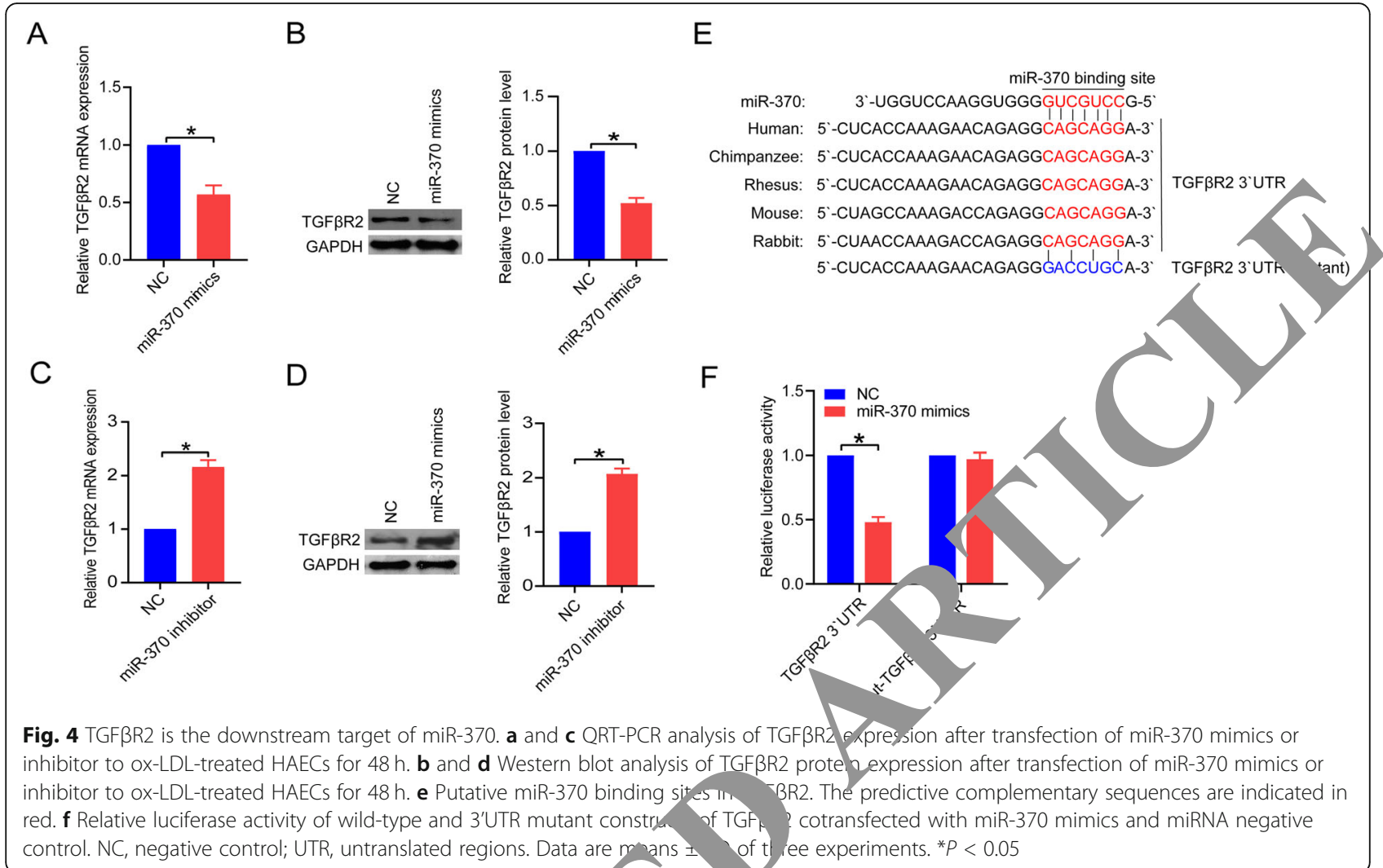

high expression of TGF $\beta 2$ with miR-370 mhis $r$ (Fig. 4c and d). Figure 4e represented the por tial bina ing sites of wild type or mutant TGF $\beta$ R? with R-370. Additionally, the luciferase activity of wild type TyF $\beta$ R2 3'UTR was evidently decreased in mi -370 mimic group, However, the luciferase activity o vutar t TGF $\beta$ R2 3'UTR remained unchanged a " transiection of miR370 mimic (Fig. 4f).

Circ_0003204/miR0-3 axi hlocks_ HAEC viability, migration and tub form, "on va downstream TGF $\beta$ R2/ phosph-SMAD?

To further exploit functionality of miR-370/TGF $\beta$ R2 axis in $\mathrm{K}$-LDL-treat, d HAECs, we performed function experin. to, such as cell viability, motility and tube for$\mathrm{ma}^{+\cdot} \eta$. It $\mathrm{s}$ aemonstrated that the inhibitory effects f $\mathrm{m}$-370 nockdown on cell proliferation (Fig. 5a), ce nucurcy (Fig. 5b and c) and capillary-like formation (Fig. di) were reversed by cotransfection of TGF $\beta$ R2 siRNA. Subsequently, we detected phosph-SMAD3 protein expression in ox-LDL-treated HAECs after transfection of TGF $\beta$ R2 siRNA. Western blot analysis displayed the decreased expression of phosph-SMAD3 after delivery of TGF $\beta R 2$ siRNA into ox-LDL-treated HAECs (Fig. 5e). Then, SIS3, a specific phosph-SMAD3 inhibitor, was used to reveal that downregulation of phosph-SMAD3 protein evidently alleviated the
Impaired proliferation (Fig. 5f), migration (Fig. 5g and h) and capillary network (Fig. 5i) of HAECs with ox-LDL stimulation whereas inhibited these angiogenic responses of HAECs with control treatment. Next, we examined the protein expression of TGF $\beta 22$ and its downstream phosph-SMAD3 in ox-LDL-treated HAECs after cotransfection with circ_0003204 siRNA and miR370 inhibitor (Fig. 5j), indicating that knockdown of circ_0003204 could inhibit the expression of TGF $\beta R 2$ and phospho-SMAD3 protein via counteracting miR-370 inhibitor function. Moreover, knockdown of circ 0003204 could block the repressive effect of miR-370 inhibitor on cell viability (Fig. 5k), motility (Fig. $5 \mathrm{l}$ and $\mathrm{m}$ ) and tube formation (Fig. 5n). Based on previous report that SMAD3 phosphorylation and nuclear translocation could be promoted by ox-LDL treatment, leading to deactivation of endothelial cell [31], our results suggested that circ_0003204 could exacerbate deactivation of HAECs in response to ox-LDL via miR370/TGFßR2/phospho-SMAD3 axis.

\section{Circ_003204 and miR-370 expression in the plasma EVs from the patients with cerebral atherosclerosis}

Based on LiftOver tool in UCSC genome browser and CIRCpedia v2 database (http://www.picb.ac.cn/rnomics/ circpedia/), there is no corresponding circRNA in the orthologous locus of mouse USP36 gene within 5-nt 


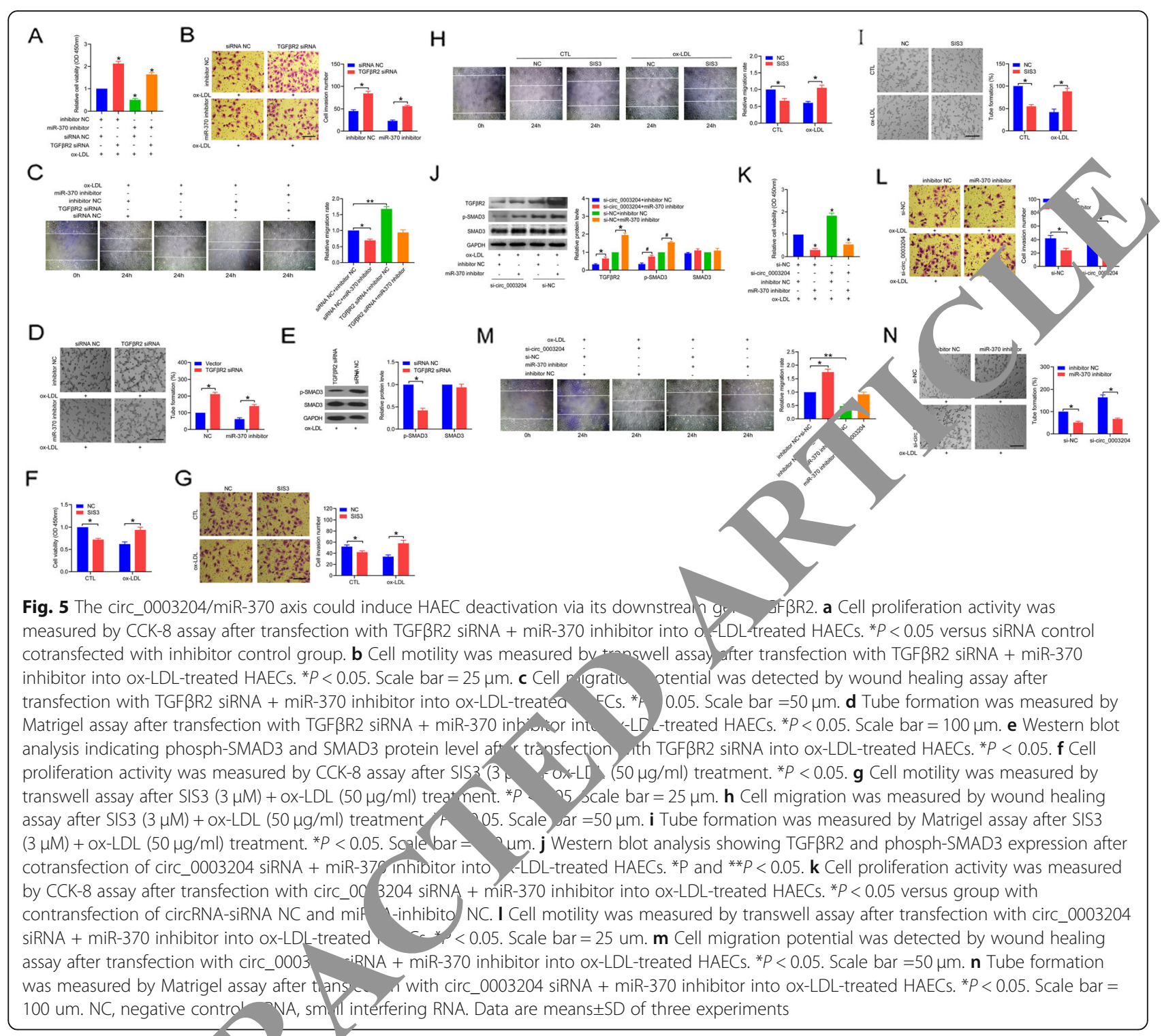

difference for ho_ch 0003204. Consequently, the expression of $n$ se-deriv a circRNA analogous to hsa circ_0003204 was detectable in mouse tissue preventing studies in mous models, such as middle cerebral artery 11 .ion Therefore, we further explore circ $000^{\circ} 04$ a $m / R-370$ level in the clinical patients. In e p sent sudy, 35 patients with cerebral atherosclero. anu 02 control subjects were recruited into our stua, Their demographic and clinical characteristics are provided in Additional file 1: Table S2. Since exosomes were demonstrated to play an essential role in cell-cell communication under various disease conditions, including atherosclerosis [29], we purified exosomes from the plasma of recruited subjects for detecting circ 0003204 and miR-370 expression. Most of the collected vesicles were typical in size as exosomes ranging from $50 \mathrm{~nm}$ to $150 \mathrm{~nm}$ (Fig. 6a), but some particles in diameter of $<50 \mathrm{~nm}$ or $>150 \mathrm{~nm}$ were also detected. Hence, these isolated vesicles were termed EVs rather than exosomes. Furthermore, these vesicles expressed characteristic exosome markers, such as FLOT-1 and CD63 (Fig. 6b). As anticipated from our results in vitro experiments, the expression of circ_0003204 in EVs from cerebral atherosclerosis patients was markedly higher compared with non-atherosclerosis groups $(P<0.05)$ (Fig. 6c). As for miR-370, it was found that the expression of miR-370 in EVs was lower in cerebral atherosclerosis patients compared with control group $(0.91 \pm 0.39$ vs $1.14 \pm 0.51$ ), although no significant difference existed between them $(P=0.54)$ (Fig. $6 \mathrm{~d})$. To explore whether HAECs secreted EVs containing circ_0003204, we purified and isolated EVs from HAEC culture medium. The size distribution of EVs was detected using Nanosight, with a size peak of $103 \mathrm{~nm}$ (Fig. 6e). A western blot for exosome 


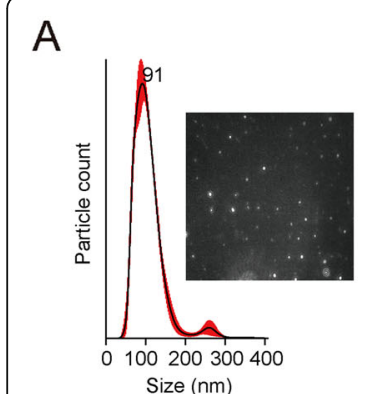

E

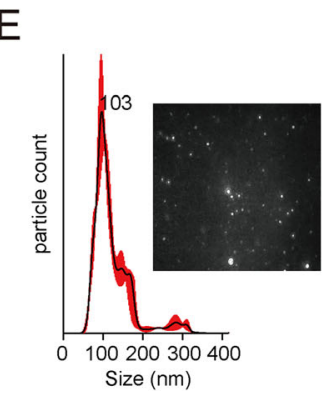

B

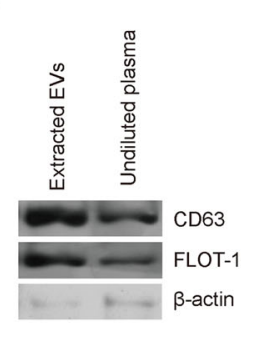

$\mathrm{F}$

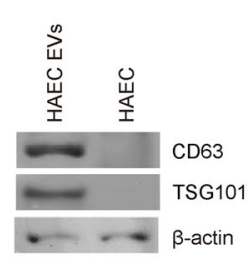

C

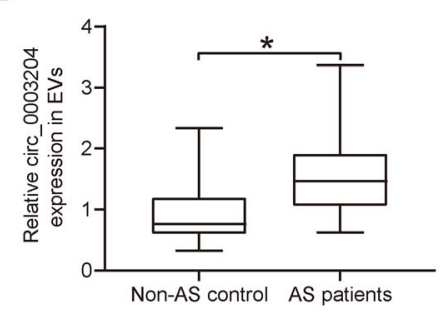

G

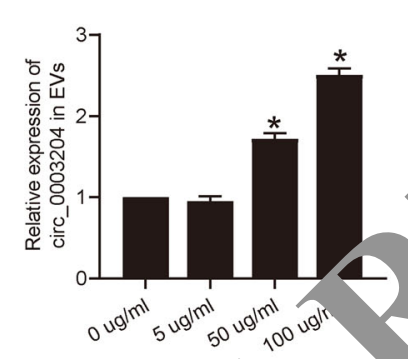

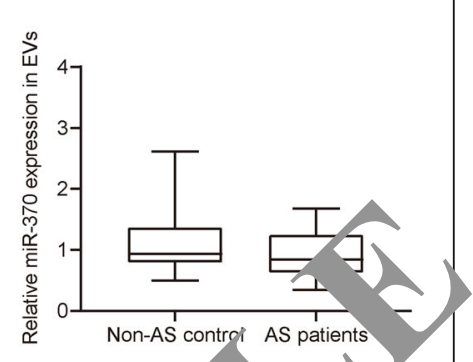

$\mathrm{H}$

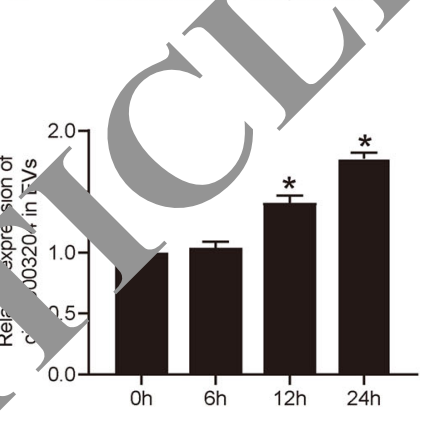

Fig. 6 Expression level of circ_0003204 and miR-370 in EVs for the cerebral atherosclerd tients and healthy controls. a The size distribution of isolated EVs measured by nanoparticle tracking analysis. b Immunoblotting of EV marker. us.ng, riti-CD63 and anti-FLOT-1 and $\beta$-actin as a loading control. c The circ_0003204 expression in EVs for cerebral atherosclerosis patients and healthy control as measured by $q$ RT-PCR. ${ }^{*} P<0.05$. d The expression level of miR-370 in EVs from cerebral atherosclerosis pati nealthy control. e Size distribution histogram of cell medium EVs by nanoparticle tracking analysis. f Immunoblotting of cell medium v for C. TSG101 and $\beta$-actin. g QRT-PCR analysis of circ_0003204 expression in the cell medium EVs at the different concentration of ox-LL $0-100 \mathrm{~g} / \mathrm{ml}$ ). ${ }^{*} P<0.05$. $\mathbf{h}$ QRT-PCR analysis of circ_0003204 expression in the EVs collected from HAECs with ox-LDL treatme $n\left(50 \mu \mathrm{g} / \mathrm{r}+\mathrm{t} / \mathrm{ne}\right.$ indicated time points. ${ }^{*} P<0.05$. Data are means $\pm \mathrm{SD}$ of three experiments

markers, such as CD63 and TSG101, furtner o firmed EVs identity (Fig. 6f). Importantly, it vas found ti at the circ_0003204 level in EVs showed creasing tendency after HAECs were exposed to differe conc ntration of

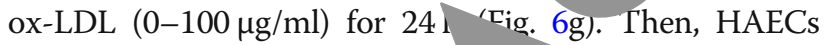
were treated with ox-LDL stipu $1,(50 \mu \mathrm{g} / \mathrm{ml})$, and qRT-PCR assay showed incre sed expression of circ 0003204 in EVs coll ted $\mathrm{nm}$ culture medium at the indicated time points.

The correlation of 0003204 expression in plasma EV and LDL- level with, erebral atherosclerosis

As incil $d$ in Additional file 1: Table S3, we found that cirr ${ }^{-032}$ expression in the plasma EVs was posi- vely elated with cerebral atherosclerosis $(r=0.469, P<$ 0. (1.r. 7a) and LDL-C level $(\mathrm{r}=0.299, P=0.014)$ (Fig. $)$. Multivariate regression analysis revealed that both circ_0003204 in the plasma EVs and LDL-C level were correlated with cerebral atherosclerosis after adjusted for gender, age, history of drinking and smoking, TC, HDL-C, TG, hypertension, diabetes, HCY, Lp-PLA2 and miR-370 expression (Additional file 1: Table S4). In addition, ROC analysis was performed to predict the impact of circ_0003204 in the plasma EVs and LDL-C level on cerebral atherosclerosis. The AUC of circ
0003204 in the plasma EVs was 0.770 (95\% CI $0.651-$ 0.890; $P<0.001$ ) (Fig. 7c), and the AUC of LDL-C level was 0.851 (95\% CI $0.761-0.952 ; P<0.001$ ) (Fig. $7 d$ ). Notably, the AUC of the combination of circ_0003204 in the plasma EVs and LDL-C level was 0.875 (95\% CI $0.777-0.954 ; P<0.001$ ) (Fig. 7e), suggesting that the combination of both had superior efficiency for predicting cerebral atherosclerosis than either circ_0003204 or LDL$\mathrm{C}$ alone. To evaluate the association of circ_0003204 level in plasma EVs with the prognosis of recruited subjects, the patients with cerebral atherosclerosis and their healthy control were respectively divided into two groups on the basis of median value of circ_0003204 level: circ_0003204 high expression and circ_0003204 low expression. Kaplan Meier analysis illustrated that cerebral atherosclerosis patients with circ_0003204 high expression developed more frequent occurrence of end events compared with those with circ_0003204 low expression (log-rank $P=$ 0.031) (Fig. 7f), but for these healthy control, the occurrence of end events had no significant difference between the subjects with circ_0003204 high expression and ones with circ_0003204 low expression (log-rank $P=0.197$ ) (Fig. 7g). This may suggest that circ_0003204 in plasma EVs represented a prognostic factor for these events appearing in cerebral atherosclerosis patients. 


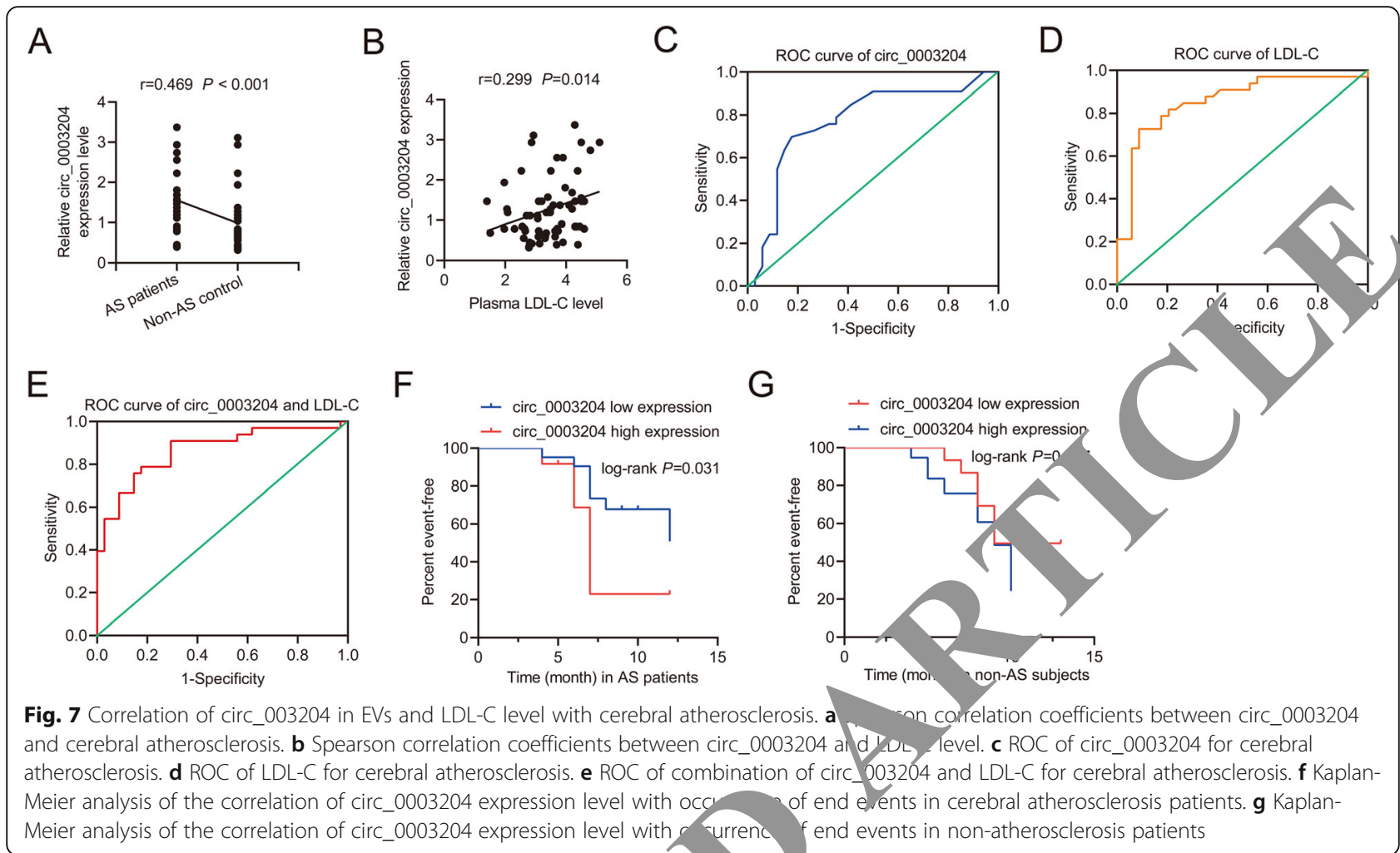

\section{Discussion}

Atherosclerosis triggered by multiple risky facor co $\mathrm{d}$ cause severe cardio/cerebral-vascular dise $\mu_{\text {such à }}$ coronary heart disease, stroke and perinera scular disease. Aberrant differentiation and phenotypic ransformation of EC are the critical initi steps contributing to atherosclerosis related vasculopath 22]. Therefore, it is important to identify ear hiomarkers predicting endothelial differentiation and $\mathrm{pl}$ in atherosclerosis for $\mathrm{r}$ ntion and treatment of such diseases. CircRNAs ner from exonic or intronic sequences are not only. indant in body fluids, but have more stable cir ar struc re which is resistant to RNA exonuclease [13]. se characteristics provide circRNA better prospect for bi marker of human disease. Importantly, a c ing amount of evidences have illustrated the socic n petween circRNAs expression and athrosc rosis progress [21, 33], but little known is about th. OIe $v$ circRNA in endothelial phenotype involved in athe Clerosis. In this study, we verified increased expression of circ_0003204 in ox-LDL treated HAECs as well as EVs from HAEC culture media and plasma of the patients with cerebral atherosclerosis. Importantly, our study elucidated the involvement of circ_0003204miR-370-TGF $\beta R 2$ axis in atherosclerosis, especially, its role in endothelial phenotype alternation, suggesting that circ_0003204 might act as a therapeutic target for oxLDL-induced ECs aberrant functions.
Differently expressed circRNAs have been reported to have important functions in buildup of atherosclerotic plaque [34]. For instances, as microRNA sponge, circCHFR, identified as a transcript at the locus of chromosome 12, serves as a stimulus for proliferation and migration of ox-LDL-induced VSMCs via sponging miR-370 [16]. Also, it was confirmed that circ_0044073 could favor proliferation and invasion of HUVECs and VSMCs by targeting miR-107 and activating its downstream JAK/STAT pathway against atherosclerosis [15]. Silencing of circWDR77 could alleviate proliferation and migration of high glucose induced VSMCs through miR-124/FGF2 axis [35]. As RBP, circANRIL was reported to induce apoptosis and alleviated proliferation of VSMC and macrophage via interaction with multiple RBPs for supervising pre-rRNA maturation and nucleolar stress [11]. In addition, Bazan HA et al. [36] discerned that the ratio of serum circR_284: miR221 is significantly elevated in the early stage of carotid plaque rupture, implying its potential as diagnostic biomarker for carotid plaque rupture and stroke. In this current study, our results revealed elevated circ 0003204 expression in ox-LDL-treated HAECs as well as in EVs secreted from ox-LDL-treated HAECS and the plasma of cerebral atherosclerosis patients, which were partly line with previous study despite different EC types. Also, we demonstrated that circ_0003204 was not only characterized as an independent risky factor 


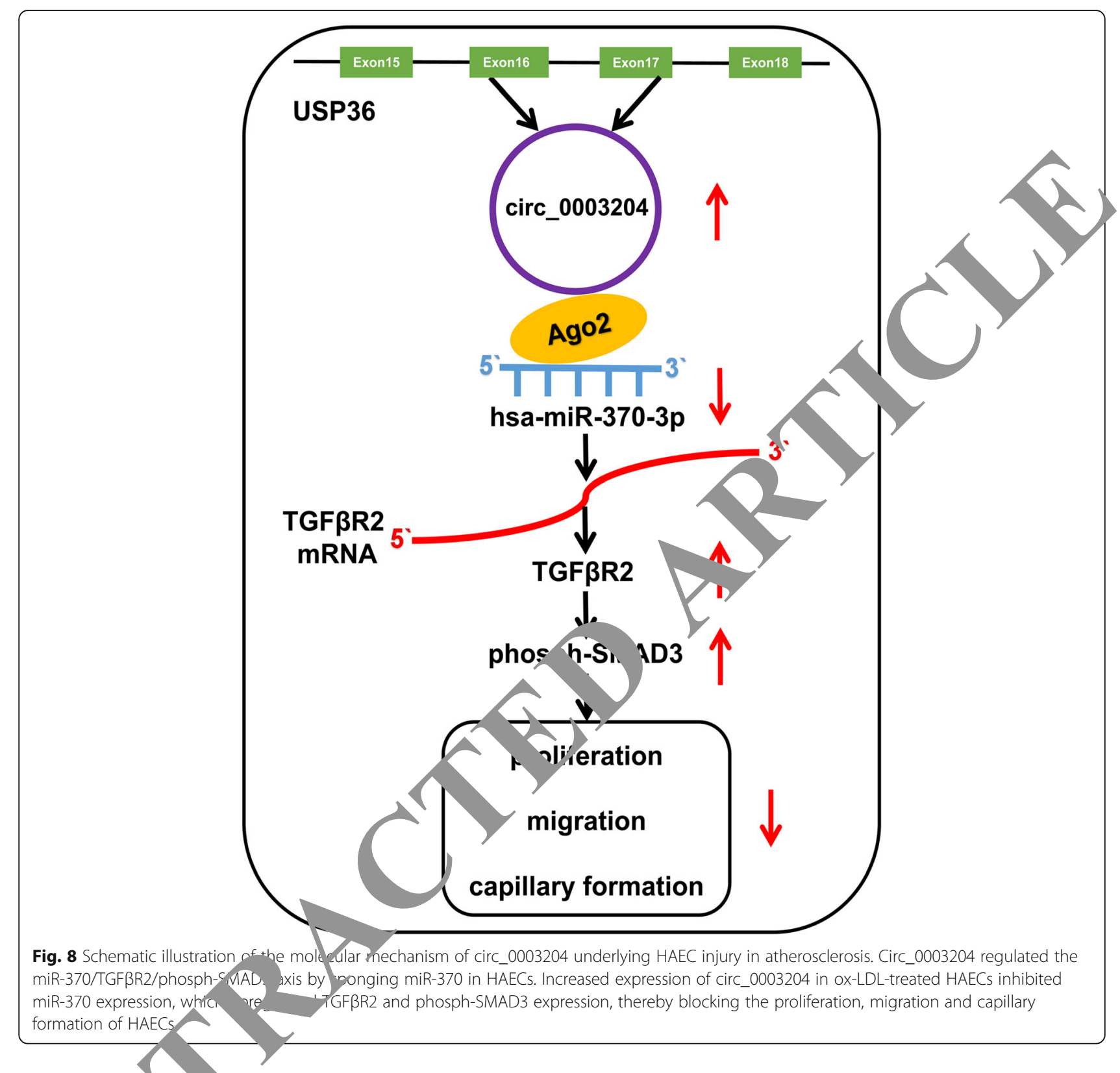

for athe c.usos pathogenesis, but had potential diagno valu or cerebral atherosclerosis. Additionally, it as und that cerebral atherosclerosis patients with h. er cspression of circ_0003204 were more likely to suffo nd events. To our best knowledge, our study is the first to verify that circ_0003204 was a promising biomarker for assessing the diagnosis and prognosis of cerebrovascular atherosclerosis and stenosis. Larger cohort studies are required to further validate the relationship between circ_0003204 level and severity of cerebral vascular stenosis.

MiR-370, which was found to interact with circ 0003204 in our study, has been demonstrated to be an important microRNA in lipid metabolism which inhibits the expression of the carnitine palmitoyl transferase $1 \alpha$ gene regulating fatty acid oxidation [37]. Recent evidences have confirmed higher expression level of miR370 in the plasma of hyperlipidemia patients with CAD as well as in the peripheral blood mononuclear cell of patients with coronary atherosclerosis [38]. Additionally, miR-370 was reported to modulate production of inflammatory factors (IL-6, IL-1 $\beta$, et al.) and oxidative stress via targeting TLR4 in ox-LDL-incubated THP-1 cells [25]. However, few studies have assessed the role of miR-370 in the endothelial injury in atherosclerosis plaque. Similar with previous findings [16], bioinformatic 
analysis in the present study uncovered that miR-370 was predicted to be involved in the development and progression of atherosclerosis plaque. Subsequently, our in vitro experiment data indicated that miR-370 expression was decreased in HAECs treated with ox-LDL and that upregulation of miR-370 expression significantly promoted cellular proliferation, migration and capillary formation. Interestingly, we also found that miR-370 level was lower in the plasma EVs from cerebral atherosclerosis patients versus control subject in spite of no significant change observed, which was inconsistent with our in vitro results and previous studies [38]. The inconsistent findings about the association between miR-370 expression and atherosclerosis may be explained as follow: (1) discrepant clinical characters and experimental samples of patients among different studies. Our recruited population was a cohort of patients with cerebral vascular stenosis $\geq 50 \%$ due to atherosclerosis, irrespective of coronary artery atherosclerosis while other studies focused on CAD patients. In addition, in our study, miR-370 level was examined in purified EVs rather than in the whole plasma or serum. (2) different sample size among studies. Therefore, further studies are required to detect miR-370 level in EVs purified from larger cohort and elucidate the role of miR-370 in EVs.

Subsequently, to confirm whether miR-370 acted as a regulator for circ_0003204-related EC phenotyp/ ird 0003204 OE vector with miR-370 mimics wer cotr. fected into ox-LDL-treated ECs. We found overe
pression of miR-370 counteracted aberant cr. EC phenotype circ_0003204 induced These resul,o suggested that miR-370, as a down ream target circ 0003204 sponged, was involved in en helia/ phenotype mediated by circ_0003204. Als יur stuay indicated that miR-370 and its targeted gene $[G /,-$ had notable impact on endothelial notyse in atherosclerosis. TGF $\beta 2$, as one of ee mes of TGF $\beta$ receptors, can bind TGF $\beta$ ligand ay a wosphorylation for cooperating with TGF 1 which ons out phosphorylation and activation of dow tream SMAD2 and SMAD3 [39]. Phospho ylated SMA_L2/SMAD3 combine with SMAD4 protein ing hetero-oligomeric complexes, which trar-acate ints nuclear to change transcription level of ulti le getres, affecting biological characteristics of CI nIc nulammation, tumor and autoimmune reactions [40- For instance, activated TGF $\beta 2$ could aggravate renal fibrosis by upregulating the expression of collagen 1 and $\alpha$-smooth muscle actin [43]. In our study, we identified a new regulatory mechanism underlying TGFßR2 which acted as a downstream target of circ 0003204. Our research demonstrated that TGF $\beta$ R2 played a pivotal role in EC proliferation, migration and capillary formation through the regulation of p-SMAD3, which positively supported our hypothesis that circ
003204/miR-370/TGFßR2 axis could regulate ectopic EC phenotype ox-LDL caused.

Several limitations should be noted in our study. Firstly, the detailed mechanism by which circ 0003204-miR-370-TGF $\beta$ R2/phosph-SMAD3 axis mediates endothelial phenotype, especially which lownstream targeted genes were directly or idirectly influenced by this axis, needs to be further val ea in future studies. Secondly, due to no conserved pro ny for circ_0003204 between species, the pressi n and function of circ_0003204 involved in the velopmental and pathological angiogenesis were unable to be examined in mouse model. Ir a tion, the main cell origin for circ_0003204 in P ma was required to explore further, althou $\mathrm{n}$ our sent results revealed that circ_0003204 w s s eased i/rto EVs in the conditioned medium of $O x-L L$ treated HAECs. Our ongoing experinen will mainly focus on the comparison o level among plasma neuron-derived $\mathrm{L}$ glia cell-derived EVs, astrocytederived endo helial cell-derived exosomes and platelet-deriyea EVs. Lastly, the number of recruited subjects was limited, and thus circ_0003204, as a biomia for diagnosis and prognosis of cerebrovascular ther sclerosis and stenosis should be further con- ned in other population groups, and the diagnostic and therapeutic value of EVs, especially miRNAs in $\mathrm{EVs}$, for cerebrovascular atherosclerosis is required to be explored in large sample size.

\section{Conclusion}

Summarily, our study disclosed regulatory mechanism of circ_0003204 that mediates endothelial phenotype through targeting miR-370-3p/TGF $\beta$ R2/phosph-SMAD3 pathway (Fig. 8). Blockage of circ_0003204 is regarded as a potential therapeutic target for alleviation of EC aberrant phenotype in atherosclerosis pathology.

\section{Supplementary information}

Supplementary information accompanies this paper at https://doi.org/10. 1186/s12929-019-0595-9.

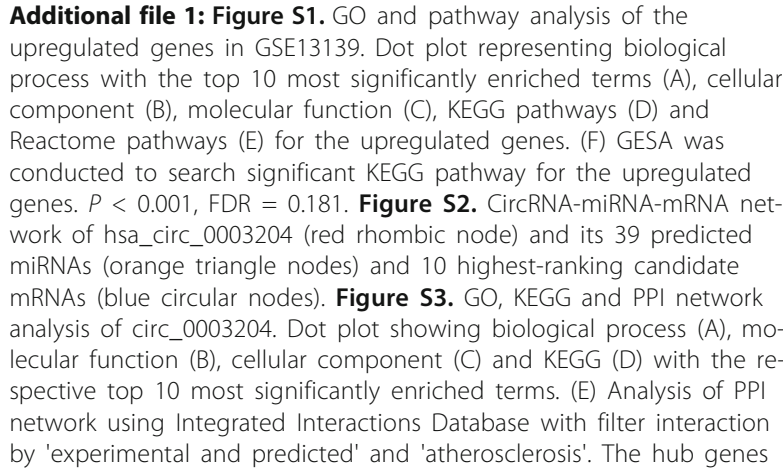
upregulated genes in GSE13139. Dot plot representing biological process with the top 10 most significantly enriched terms (A), cellular component (B), molecular function (C), KEGG pathways (D) and Reactome pathways (E) for the upregulated genes. (F) GESA was conducted to search significant KEGG pathway for the upregulated genes. $P<0.001, F D R=0.181$. Figure S2. CircRNA-miRNA-mRNA network of hsa_circ_0003204 (red rhombic node) and its 39 predicted miRNAs (orange triangle nodes) and 10 highest-ranking candidate mRNAs (blue circular nodes). Figure S3. GO, KEGG and PPI network analysis of circ_0003204. Dot plot showing biological process (A), mo lecular function (B), cellular component (C) and KEGG (D) with the respective top 10 most significantly enriched terms. (E) Analysis of PPI network using Integrated Interactions Database with filter interaction by 'experimental and predicted' and 'atherosclerosis'. The hub genes 
in PPI was labeled with the colored nodes of which the color depth referred to the 'degree' calculated by Cytoscape. (F) KEGG enriched cluster of genes from PPI network using Metascape. (G) The genes in Fig.F were colored by their P-value. $(H)$ The enriched cluster showing interaction of 7 modules in PPI network as analyzed by Metascape. (1) Seven modules obtained from PPI network. Figure S4. The expression level of mmu-miR-370 and mmu-miR-197 in GSE34645, GSE34644 and GSE34646. (A) A microarray heat map from GSE34645 representing discrepant miRNA expression in atherosclerosis plaque on 3 month high fat diet compared with undieased arterial tissue $(|\log 2 \mathrm{FC}|$ $>2, P<0.05$ ). mmu-miR-370 was marked by red. (B), (C) and (D) Volcano plots presenting the differently expressed miRNAs from GSE34645, GSE34644 and GSE34646. Both mmu-miR-370 and mmumiR-197 were indicated by blue arrow. Figure S5. Involvement of TGF $\beta R 2$ in FOXO signaling pathway.

\section{Abbreviations}

AUC: Area under ROC curve; CAD: Coronary artery disease: ceRNA: Competing endogenous RNA; circRNAs: Circular RNAs; DM: Diabetes mellitus; ECs: Endothelial cells; EVs: Extracellular vesicles; FDR: False discovery rate; FISH: Fluorescence in situ hybridization; GEO: Gene Expression Omnibus; GO: Gene Ontology; GSEA: Gene set enrichment analysis; HAECs: Human aorta endothelial cells; HDL-C: High-density lipoprotein cholesterol; HUVEC: Human umbilical vein endothelial cell; KEGG: Kyoto Encyclopedia of Genes and Genomes; LDL-C: Low-density lipoprotein cholesterol; ncRNAs: Non-coding RNAs; NTA: Nanoparticle tracking analysis; OE: Overexpresson; PBS: Phosphate buffered saline; PPP: Platelet-poor plasma; qRT-PCR: Quantitative real-time PCR; RBP: RNA-binding protein; RIP: RNA immunoprecipitation; ROC: Receiver operating characteristic; SD: Standard deviation; TC: Total cholesterol; TG: Triglyceride; TIA: Transient ischemic attack; UTR: 3' Untranslated region; VSMC: Vascular smooth muscle cell

\section{Acknowledgements}

Not applicable.

\section{Authors' contributions}

SZ conceived the project and designed the research. SZ and GS ducted the experiments. SQ, YY and SX analyzed the data. JY, XX and ZS Co red clinical samples. AW performed the manuscript rey sion. All authors read and approved the final manuscript.

\section{Funding}

This work was supported by grants from th Shandong Province, China (No.ZR2016HP04, N .ZRz, 19MH062) and the National Natural Science Foundare f Chin (No.81601020).

Availability of data and mate

All data used during the current s available from the corresponding author on reasonab e est.

\section{Ethics approval and conse. to participate}

This stud. Shandong "Clat Q foshan Hospital, Shandong University, and patient con was ac prior to the initiation of experiment.

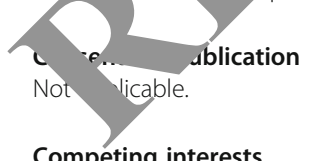

\section{Competing interests}

The authors declare that they have no competing interests.

Received: 21 July 2019 Accepted: 18 November 2019 Published online: 03 January 2020

\section{References}

1. Uchida Y, Maezawa Y, Uchida Y, Hiruta N, Shimoyama E, Kawai S. Localization of oxidized low-density lipoprotein and its relation to plaque morphology in human coronary artery. PLoS One. 2013;8:e55188.
2. Poston RN. Atherosclerosis: integration of its pathogenesis as a selfperpetuating propagating inflammation: a review. Cardiovasc Endocrinol Metab. 2019;8:51-61.

3. Pirillo A, Bonacina F, Norata GD, Catapano AL. The interplay of lipids, lipoproteins, and immunity in atherosclerosis. Curr Atheroscler Rep. 2018;20:12.

4. Tang F, Yang TL. MicroRNA-126 alleviates endothelial cells injury in atherosclerosis by restoring autophagic flux via inhibiting of PI3K/Nu'mTOR pathway. Biochem Biophys Res Commun. 2018;495:1482-9.

5. Qiu J, Wang G, Zheng Y, Hu J, Peng Q, Yin T. Coordination of migration. Ann Biomed Eng. 2011;39:2869-78.

6. Badimon L, Vilahur G. Thrombosis formation on plaque rupture. J Intern Med. 2014;276:618-32

7. Tousoulis D, Oikonomou E, Economou EK, C ea F, Kaski JC. , ammatory cytokines in atherosclerosis: current therap tic approaches. Eur Heart J. 2016;37:1723-32.

8. Feinberg MW, Moore KJ. MicroRNA. Jation, 2016;118:703-20.

9. Gorur A, Celik A, Yildirim DD undes A, Ta $\measuredangle$. Investigation of possible effects of microRNAs inv vea vulation of lipid metabolism in the pathogenesis of atherosclerosis. $N \quad$ Bjol Rep. 2019;46:909-20.

10. Jiang $L$, Qiao $Y$, War $y<. \quad X$, Wang $H, 4 J$. Inhibition of microRNA-103 attenuates inflammation an ndopl mic reticulum stress in atherosclerosis through disrupting the PTE ák signaling. J Cell Physiol. 2020;235:380-93.

11. Holdt LM, Stahringer Sass K, Pichler G, Kulak NA, Wilfert W, Kohlmaier A Herbst A a r off BH, colaou A, Gabel G, Beutner F, Scholz M, Thiery J, Musunur I , _ KMann M, Teupser D. Circular non-coding RNA ANRIL modulates roson, al RNA maturation and atherosclerosis in humans. Nat Commun. 2016;7:12429.

"rou T, Chen X. Long intergenic noncoding RNA p21 mediates oxidized nduced apoptosis and expression of LOX1 in human coronary artery en thelial cells. Mol Med Rep. 2017;16:8513-9.

Hoidt LM, Kohlmaier A, Teupser D. Molecular roles and function of circular RNAs in eukaryotic cells. Cell Mol Life Sci. 2018;75:1071-98. Greene J, Baird AM, Brady L, Lim M, Gray SG, McDermott R, Finn SP. Circular RNAs: Biogenesis, Function and Role in Human Diseases. Front Mol Biosci. 2017;4: 38.

15. Shen L, Hu Y, Lou J, Yin S, Wang W, Wang Y, Xia Y, Wu W. CircRNA0044073 is upregulated in atherosclerosis and increases the proliferation and invasion of cells by targeting miR107. Mol Med Rep. 2019;19:3923-32.

16. Yang $L$, Yang F, Zhao $H$, Wang $M$, Zhang Y. Circular RNA circCHFR facilitates the proliferation and migration of vascular smooth muscle via miR-370/ FOXO1/Cyclin D1 pathway. Mol Ther Nucleic Acids. 2019;16:434-41.

17. Li CY, Ma L, Yu B. Circular RNA hsa_circ_0003575 regulates oxLDL induced vascular endothelial cells proliferation and angiogenesis. Biomed Pharmacother. 2017:95:1514-9.

18. Gao J, Yang S, Wang K, Zhong Q, Ma A, Pan X. Plasma miR-126 and miR-143 as potential novel biomarkers for cerebral atherosclerosis. J Stroke Cerebrovasc Dis. 2019;28:38-43.

19. Powers WJ, Derdeyn CP, Biller J, Coffey CS, Hoh BL, Jauch EC, Johnston KC, Johnston SC, Khalessi AA, Kidwell CS, Meschia JF, Ovbiagele B, Yavagal DR. 2015 American Heart Association/American Stroke Association focused update of the 2013 guidelines for the early Management of Patients with Acute Ischemic Stroke Regarding Endovascular Treatment: a guideline for healthcare professionals from the American Heart Association/American Stroke Association. Stroke. 2015;46:3020-35.

20. Zhao L, Yu J, Wang J, Li H, Che J, Cao B. Isolation and identification of miRNAs in exosomes derived from serum of colon cancer patients. J Cancer. 2017:8:1145-52.

21. Bayoumi AS, Aonuma T, Teoh JP, Tang YL, Kim IM. Circular noncoding RNAs as potential therapies and circulating biomarkers for cardiovascular diseases. Acta Pharmacol Sin. 2018;39:1100-9.

22. Zhou Y, Cao ZQ, Wang HY, Cheng YN, Yu LG, Zhang XK, Sun Y, Guo XL. The anti-inflammatory effects of Morin hydrate in atherosclerosis is associated with autophagy induction through CAMP signaling. Mol Nutr Food Res. 2017;61:1600966.

23. Mitra A, Pfeifer K, Park KS. Circular RNAs and competing endogenous RNA (ceRNA) networks. Transl Cancer Res. 2018;7:S624-8.

24. Ou H, Liu C, Feng W, Xiao X, Tang S, Mo Z. Role of AMPK in atherosclerosis via autophagy regulation. Sci China Life Sci. 2018;61:1212-21. 
25. Tsuchiya K, Tanaka J, Shuiqing Y, Welch CL, DePinho RA, Tabas I, Tall AR, Goldberg IJ, Accili D. FoxOs integrate pleiotropic actions of insulin in vascular endothelium to protect mice from atherosclerosis. Cell Metab. 2012;15:372-81.

26. Tian D, Sha Y, Lu JM, Du XJ. MiR-370 inhibits vascular inflammation and oxidative stress triggered by oxidized low-density lipoprotein through targeting TLR4. J Cell Biochem. 2018;119:6231-7.

27. Zhang $H$, Sun $X$, Hao D. Upregulation of microRNA-370 facilitates the repair of amputated fingers through targeting forkhead box protein 01. Exp Biol Med (Maywood). 2016;241:282-9.

28. Menghini R, Casagrande V, Cardellini M, Ballanti M, Davato F, Cardolini I, Stoehr R, Fabrizi M, Morelli M, Anemona L, Bernges I, Schwedhelm E, Ippoliti A, Mauriello A, Boger RH, Federici M. FoxO1 regulates asymmetric dimethylarginine via downregulation of dimethylaminohydrolase 1 in human endothelial cells and subjects with atherosclerosis. Atherosclerosis. 2015;242:230-5.

29. McCaffrey TA. TGF-betas and TGF-beta receptors in atherosclerosis. Cytokine Growth Factor Rev. 2000;11:103-14.

30. Nickel J, Ten DP, Mueller TD. TGF-beta family co-receptor function and signaling. Acta Biochim Biophys Sin Shanghai. 2018;50:12-36.

31. Blaser MC, Aikawa E. Roles and regulation of extracellular vesicles in cardiovascular mineral metabolism. Front Cardiovasc Med. 2018:5:187.

32. Gimbrone MJ, Garcia-Cardena G. Endothelial cell dysfunction and the pathobiology of atherosclerosis. Circ Res. 2016;1 18:620-36.

33. Ding $S$, Zhu $Y$, Liang $Y$, Huang $H, X u Y$, Zhong C. Circular RNAs in vascular functions and diseases. Adv Exp Med Biol. 2018;1087:287-97.

34. Zhang F, Zhang R, Zhang X, Wu Y, Li X, Zhang S, Hou W, Ding Y, Tian J, Sun $\mathrm{L}$, Kong $\mathrm{X}$. Comprehensive analysis of circRNA expression pattern and circRNA-miRNA-mRNA network in the pathogenesis of atherosclerosis in rabbits. Aging (Albany NY). 2018;10:2266-83.

35. Chen J, Cui L, Yuan J, Zhang Y, Sang H. Circular RNA WDR77 target FGF-2 to regulate vascular smooth muscle cells proliferation and migration by sponging miR-124. Biochem Biophys Res Commun. 2017;494:126-32.

36. Bazan HA, Hatfield SA, Brug A, Brooks AJ, Lightell DJ, Woods TC. Carotio plaque rupture is accompanied by an increase in the ratio of serum ircR 284 to miR-221 levels. Circ Cardiovasc Genet. 2017;10:e001720.

37. Iliopoulos D, Drosatos K, Hiyama Y, Goldberg IJ, Zannis VI. Mic OnNA-3x, controls the expression of microRNA-122 and Cpt1alpha ar ffects lipid metabolism. J Lipid Res. 2010;51:1513-23.

38. Hoekstra M, van der Lans CA, Halvorsen B, Gullestad I Kuliper J, Us, P, van Berkel TJ. The peripheral blood mononuclear cell microRNA sig ature of coronary artery disease. Biochem Biophys Re Commun. 2010;394:792-7.

39. Lampropoulos P, Zizi-Sermpetzoglou A, Rizos S, takis A, N kiteas N, Papavassiliou AG. Prognostic significance of trans beta (TGF-beta) signaling axis molecule d F-cadherin in colorectal cancer. Tumour Biol. 2012;33:1005-14.

40. Liu L, Liu X, Ren X, Tian Y, Chen Z, Xu X, LY Y, Jizng C, Fang Y, Liu Z, Fan B, Zhang $Q$, Jin G, Yang $X$, Zhang , mad2 and,Smad3 have differential sensitivity in relaying $T G$ sic lina and inversely regulate early lineage specification. Sci Rep 2016 ;

41. Ai J, Nie J, He J, G Q Q, Li M, L Viu Y, Zhou Z, Zhu F, Liang M, Cheng Y, Hou FF. GQ5 bri a ia fibrosj, in obstructive nephropathy by selectively inhibiting TGP-beta-li, ed Smad3 phosphorylation. J Am Soc Nephrol. 2015;26\% 02/ 38 .

42. Zhan Th role of transforming growth factor beta in T helper 17 differe, on. $\mathrm{mm}$ nol. 2018;155:24-35.

43. g J, L. Yhar, Zhou Z, Zhang M, Li D, Zhang CY, Zen K, Liu Z. Mi. DRNA-19, a/b mitigate renal fibrosis by targeting TGF-beta receptor 2. J An phrol. 2016;27:3006-21.

\section{Publi,sher's Note}

Springer Nature remains neutral with regard to jurisdictional claims in published maps and institutional affiliations.

Ready to submit your research? Choose BMC and benefit from:

- fast, convenient online submission

- thorough peer review by experienced researchers in your field

- rapid publication on acceptance

- support for research data, including large and complex data types

- gold Open Access which fosters wider collaboration and increased citations

- maximum visibility for your research: over $100 \mathrm{M}$ website views per year

At BMC, research is always in progress.

Learn more biomedcentral.com/submissions 